\title{
Identification of differentially expressed genes associated with asthma in children based on the bioanalysis of the regulatory network
}

\author{
CHUNYAN WANG ${ }^{1}$, HENGTAO LI ${ }^{1}$, LANFANG CAO $^{2}$ and GENZAI WANG ${ }^{1}$ \\ ${ }^{1}$ Department of Pediatrics, Shanghai Fengxian Fengcheng Hospital; ${ }^{2}$ Department of Pediatrics, \\ Ren Ji Hospital Affiliated to Shanghai Jiaotong University School of Medicine, Shanghai 200000, P.R. China
}

Received November 4, 2017; Accepted May 18, 2018

DOI: $10.3892 / \mathrm{mmr} .2018 .9205$

\begin{abstract}
Asthma, the most common chronic respiratory tract disease in children, is characterized by allergy, recurring airway obstruction and bronchospasm. The aim of the present study was to screen critical differentially expressed genes (DEGs) involved in asthma in children. Gene expression in different tissues was compared between asthmatic children and healthy control subjects in order to identify DEGs associated with asthma. Protein-protein interaction (PPI) networks were constructed for the DEGs and weighted gene co-expression network analysis methods were used to further determine the functional modules associated with DEGs in different tissue samples. In addition, the gene co-expression network was constructed. Gene Ontology function analysis and pathway analysis were conducted to identify critical DEGs. The results identified numerous DEGs from the different tissue samples, including 1,662 DEGs from nasal-epithelium tissue samples, 572 DEGs from peripheral blood (PB) samples and 146 DEGs from PB mononuclear cells samples. In the PPI network, F-box only protein 6 (FBXO6), histone deacetylase 1 (HDAC1) and amyloid $\beta$ precursor protein $(A P P)$ were hub genes and served an important role in the process of asthma. In addition, proliferating cell nuclear antigen (PCNA), integrin $\alpha-4$ (ITGA4), catenin $\alpha-1$ (CTNNA1), nuclear factor- $\kappa \mathrm{B} 1(N F-\kappa B 1)$ and mechanistic target of rapamycin $(M T O R)$ may be critical DEGs involved in the progression of asthma in children. These results suggested that $F B X O 6, H D A C 1$ and $A P P$ may interact with $P C N A, I T G A 4, C T N N A 1, N F-\kappa B 1$ and $m T O R$ in the progression of asthma in children.
\end{abstract}

Correspondence to: Professor Genzai Wang, Department of Pediatrics, Shanghai Fengxian Fengcheng Hospital, 9983 Chuannanfeng Highway, Fengxian, Shanghai 200000, P.R. China

E-mail: wang_gzai@126.com

Key words: differentially expressed genes, microarray, asthma, respiratory epithelia

\section{Introduction}

Asthma is a common chronic respiratory tract disease in children, and it affects more than 6.1 million American children in recent years (1). Minority and low-income children were reported that suffered from a higher ratio of asthma morbidity and mortality (2). Asthma is characterized by allergy, recurring airway obstruction, and bronchospasm (3). It is mainly caused by a combination of genetic and environmental factors, such as air pollution, allergens, viral infection, aspirin and beta blockers (4). Although great scientific advances have improved our understanding of asthma and promoted abilities to control asthma effectively, the mechanism of asthma is still unclear and needs to be explored. Exploring the progressive of asthma and assessing asthma severity, especially asthma in children, have been defined as urgent and persistent task to initiating appropriate therapy.

Recent studies on asthma in children have focused on genetic factors, especially critical genes and mRNAs in the pathogenesis of childhood asthma (5). $\beta 2$-AR agonist is now the most important bronchodilator for the treatment of asthma in clinical, and the polymorphism of the $A D R B 2$ response to inhaled beta-agonists in children with asthma (6). Besides, imbalance of $\mathrm{CD}^{+} \mathrm{T}$ cell subgroup were also major factors resulting in asthma, and study showed that differentiation of Th2 cell, regulatory T cells (Treg) were involved in the occurrence of asthma (7). To date, the pathogenetic basis for the relationship between potential gene expression changes and asthma has not been clearly elucidated.

In 2017, Yang et al (8) identified the DNA methylation and gene expression changes in nasal-epithelium (NE) tissue associated with childhood asthma. They demonstrated that the methylation marks in the nasal epithelia of children with allergic asthma were associated with gene expression changes. Based on the microarray data deposited by Yang et al (8), as well as other three microarray data of asthma downloaded from GEO database, we identified many differentially expressed genes (DEGs) from different tissue samples associated with asthma in children. Furthermore, we constructed protein-protein interaction (PPI) network for the DEGs and investigated the functional modules of DEGs in NE, peripheral blood mononuclear cells (PBMC) and peripheral 
blood (PB) samples. Gene Ontology (GO) functional analysis and Kyoto Encyclopedia of Genes and Genomes (KEGG) pathway enrichment analysis were performed. In addition, gene co-expression network was constructed to identify the critical DEGs in asthmatic children. Our study might provide novel diagnostic biomarkers and therapeutic target molecules in progression of asthma in children.

\section{Materials and methods}

Data source. The four microarray datasets associated with asthma in children [access nos. GSE65204 (8), GSE40732 (9), GSE40888 (10), and GSE35571 (11)] were downloaded from National Center of Biotechnology Information (NCBI) GEO database. GSE65204 dataset was sequenced on the platform of GPL14550 Agilent-028004 SurePrint G3 Human GE 8x 60K Microarray (www.ncbi.nlm.nih.gov/geo/query/acc. cgi?acc=GPL14550), including NE tissue samples from 36 asthmatic and 33 non-asthmatic children (Table I). GSE40732 dataset included PBMCs from 97 atopic asthmatic and 97 nonatopic nonasthmatic children, which was sequenced on platform of GPL16025 NimbleGen Homo sapiens Expression Array [(100718_HG18_opt_expr) www.ncbi.nlm.nih. gov/geo/query/acc.cgi?acc=GPL16025]. GSE40888 dataset was sequenced on the platform of GPL6244 (HuGene-1_0-st) Affymetrix Human Gene 1.0 ST Array, including PBMCs samples from 41 asthmatic and 24 non-asthmatic children. GSE35571 dataset contained PB samples from 60 asthmatic and 64 non-asthmatic children based on the platform of GPL570 (HG-U133_Plus_2) Affymetrix Human Genome U133 Plus 2.0 Array.

DEGs screening. The DEGs between disease groups and control groups were screened by the limma package (12) in $\mathrm{R}$ software. The adjusted $\mathrm{P}$-value $<0.05$ and $\log 2$ fold-change (FC) $\mid>1$ were considered as cut-off criteria.

The microarray data from GSE40732 and GSE40888 datasets were analyzed to identify DEGs in PBMCs samples between asthmatic children and healthy groups. Besides, GSE35571 dataset was used to identify DEGs in PB samples and GSE65204 dataset were used to identify DEGs in NE tissue samples.

Identification of co-expression modules. To identify the gene co-expression modules related to different tissue samples, we used weighted gene co-expression network analysis (WGCNA) (13) package to further mine the modules. The WGCNA provided topology properties of co-expression network, as well as the correlation of two node genes and relevant other genes. Besides, we changed the connection coefficient into weight coefficient and screened significant co-expression modules that related to different tissue samples using WGCNA analysis.

PPI network construction. For the identified DEGs, we screened the PPI relationship pairs of DEGs from some common databases. The databases included HPRD (www.hprd.org/), BIOGRID (thebiogrid.org/), DIP (dip.doe-mbi.ucla.edu/dip/Main.cgi), MINT (mint.bio. uniroma2.it/mint/Welcome.do), menthe (mentha.uniroma2 .it/index.php), PINA (cbg.garvan.unsw.edu.au/pina/), InnateDB (www.innatedb.com/), Instruct (instruct.yulab. org/index.html). We then obtained the regression coefficient value of each relationship pair in different conditions through linear regression analysis.

To investigate the changes of adjust power under two conditions, we proposed a method to calculate the changes of adjust power associated with screened PPI relationship pairs. The equation is as follows:

$$
\mathrm{d} D R l_{i}=\frac{C 2 \operatorname{coef}_{\mathrm{i}}-C \text { lcoef }_{i}}{\sqrt{C 2 s d_{i}^{2}+C 1 s d_{i}^{2}+m}}
$$

$\mathrm{C} 1$ coef $_{\mathrm{i}}$ and $\mathrm{C} 2$ coef $_{\mathrm{i}}$ represent regression coefficient value of each relationship pairs in condition 1 and condition 2 , respectively; $\mathrm{C} 1 s d_{\mathrm{i}}$ and $\mathrm{C} 2 c s d_{\mathrm{i}}$ represent standard deviation (SD) values responding to regression coefficient in condition 1 and condition 2, respectively; $\mathrm{M}$ represents compensation coefficient; If a relationship pair is only presented in the condition of disease, then its regression coefficients and SD value would be zero in other conditions; $\mathrm{d} D R l$ ranging from positive to negative value represents that the regulative relations are increased or decreased from condition 1 to 2 . The higher the absolute values of $\mathrm{d} D R l$, the greater change of adjust power.

In our study, we mainly compared the gene relationships in three types of tissues: NE-Normal (from normal to NE), PB-Normal (from normal to PB) and PBMC-Normal (from normal to PBMC). After calculating $\mathrm{d} D R l$ values of the whole relationships, we constructed the weighted correlation network. In the network, the $\mathrm{d} D R l$ values represented edge weight. The higher the absolute values of $\mathrm{d} D R l$, the greater significance of relationship pairs. Besides, we performed the KEGG pathway and GO analyses to identify the enrichment functions of DEGs in the network.

Identification of specific genes. In the weighted correlation network, the $\mathrm{d} D R l$ values were used to evaluate the adjusted power of DEGs. For the specific gene g, we calculated its adjusted power through the following formula:

$$
\mathrm{d} D R g_{g}=\sum_{i=1}^{k}\left|d D R I_{i}\right|
$$

$\mathrm{g}$ represents the specific genes; $\mathrm{k}$ represents the number of relationships corresponding with the specific gene; the $\mathrm{d} D R l$ can be displayed as $\mathrm{d} D R l_{\mathrm{i}}(\mathrm{i}=1,2, \ldots, \mathrm{k})$. The higher the $\mathrm{dDRg}$ values, the greater effect of the specific genes on the network. Finally, we exacted top 10 genes according to the $\mathrm{d} D R g$ value.

\section{Results}

Identification of DEGs. We identified many DEGs from different tissue samples, including 1,662 DEGs from NE tissue samples, 572 DEGs from PB samples, 146 DEGs from PBMC samples. Furthermore, the genes related to asthma were downloaded from DisGeNET database and the number of genes in three types of tissue was counted, respectively. A total of 169 DEGs related to asthma were screened from NE tissue, 38 DEGs were screened from PB and 14 DEGs were screened from PBMC (Fig. 1A).

The Venn diagram was used to visualize the DEGs screened from differential tissues (Fig. 1B). Among these genes, colony 
Table I. Microarray databases associated with asthmatic children, including GSE65204, GSE40732, GSE40888 and GSE35571.

\begin{tabular}{|c|c|c|c|c|}
\hline Accession & Platform & Tissue & $\begin{array}{c}\text { Asthmatic } \\
\text { samples (n) }\end{array}$ & $\begin{array}{l}\text { Non-asthmatic } \\
\text { samples (n) }\end{array}$ \\
\hline GSE65204 & GPL14550 & Nasal-epithelium & 36 & 33 \\
\hline GSE40732 & GPL16025 & Peripheral blood mononuclear cells & 97 & 97 \\
\hline GSE40888 & GPL6244 & Peripheral blood mononuclear cells & 41 & 24 \\
\hline GSE35571 & GPL570 & Peripheral blood & 60 & 64 \\
\hline
\end{tabular}

A

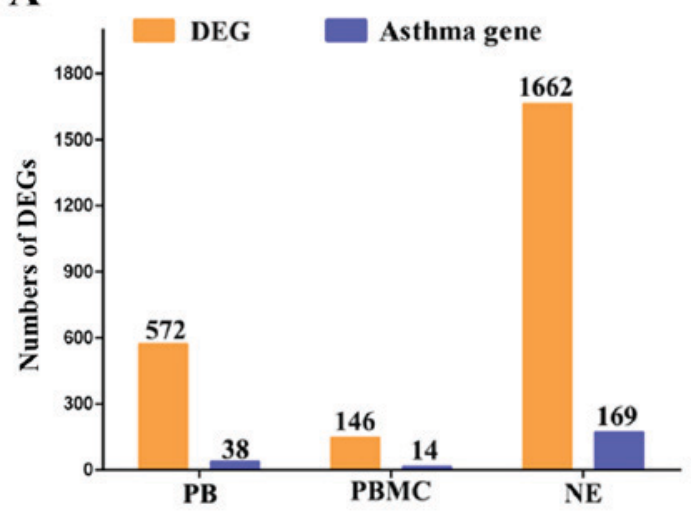

B

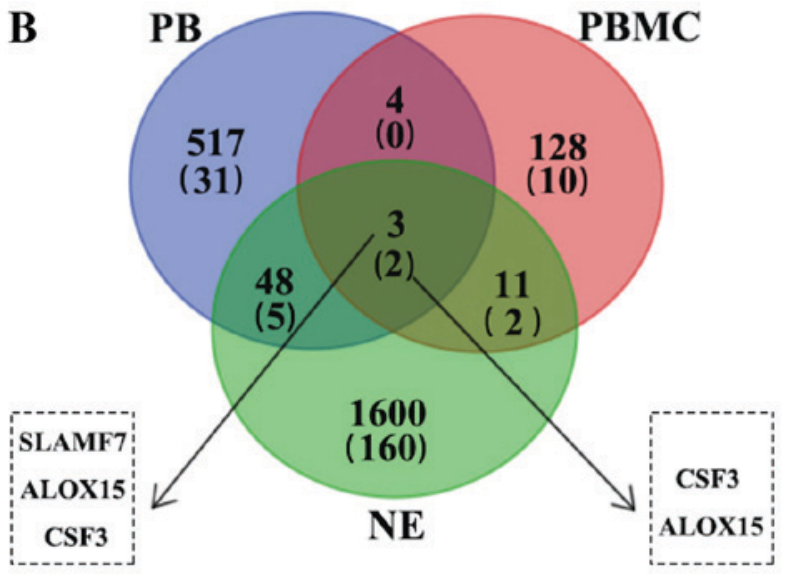

Figure 1. DEGs in three types of tissues associated with asthmatic children and the Venn diagrams. (A) The DEGs in three types of tissues associated with asthmatic children. (B) Venn diagrams presenting the number of DEGs in the different tissues of asthmatic children. DEGs, differentially expressed genes; PB, peripheral blood; PBMC, peripheral blood mononuclear cells; NE, nasal-epithelium; CSF3, colony stimulating factor 3; ALOX15, arachidonate 15-lipoxygenase; SLAMF7, SLAM family member 7.

stimulating factor 3 (CSF3) and arachidonate 15-lipoxygenase (ALOX15) were significantly differentially expressed in all three types of tissue.

PPI network analysis. The PPI relationship pairs associated with DEGs were extracted from common databases and the PPI network was constructed. To further explore the DEGs related to PBMC tissue, we take the union set of DEGs in two databases (GSE40732 and GSE40888) to construct the PPI network. In the PPI network, a total of 13496 nodes and 71275 relationship pairs were identified (Fig. 2A). F-box only protein 6 (FBXO6) and histone deacetylase 1 (HDACl) were hub genes and played an important role in the process of asthma. Additionally, we performed the topological centrality analysis of the network. The results showed that there were significance differences between the DEGs in NE and PB samples according to the degree, topological coefficient, and closeness centrality analyses. The values of degree in $\mathrm{NE}$ tissue were higher than that in PBMC tissue. The values of degree and closeness centrality in PBMC tissue were higher than that in PB tissue (Fig. 2C).

The DEGs related to asthma were downloaded from DisGeNET databases (http://www.disgenet .org/web/DisGeNET/menu/home;jsessionid=mjbtjh4yncdzuq jlnk3rnvbn) and a total of 1,403 DEGs were screened, including 104 DEGs associated with asthma in children. We integrated the PPI relationship pairs and constructed the PPI network related to asthma, including 11,960 nodes and
61,164 relationship pairs (Fig. 2B). Based on the topology properties analysis (including degree, topological coefficient, closeness centrality and betweenness centrality), we found that there was no significant difference between childhood asthma DEGs and other asthma DEGs (Fig. 2D).

Gene co-expression network analysis. The cuttee Static Color function in the WGCNA package was used to mine the functional modules associated with different tissues. The parameters were set as follows: The $\mathrm{X}$-axis represents gene degrees $\mathrm{k}$, Y-axis represents the ratio of genes $\mathrm{p}(\mathrm{k})$ (Fig. 3A). Hierarchical cluster diagrams were used to visualize the functional modules associated with different tissues (Fig. 3B). A total of 100 genes were selected randomly from network modules and the correlation between these genes were further investigated (Fig. 3C).

We analyzed the P-values of each DEG in the disease groups and normal tissues. A higher degree indicated that the DEG had greater connection with other genes and might play an important role in the progression of asthma. $\mathrm{K}$ represents the degree of each DEG in the modules. P-values represent the significance of each DEG. The correlation between $\mathrm{K}$ values and $-\log 10(\mathrm{p})$ was calculated. According to the GO and pathway analysis, we obtained the functional modules related to different tissues. Fig. 3D showed functional modules of PB tissue, and functional modules of other tissues were also obtained with the same method (data not shown).

Two functional modules in the color of brown and yellow were screened significantly associated with PB tissue; 
A

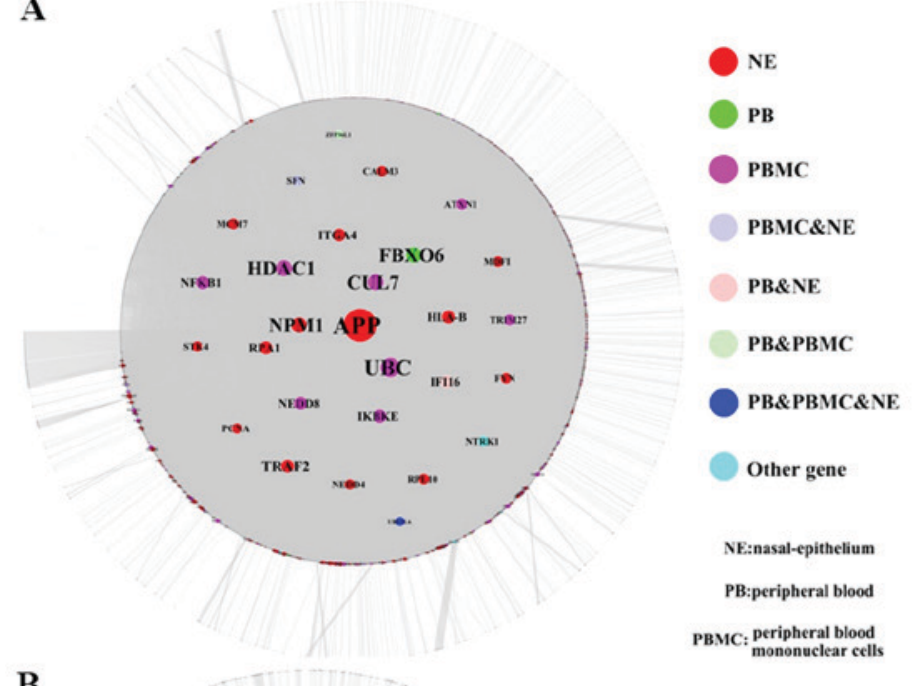

B

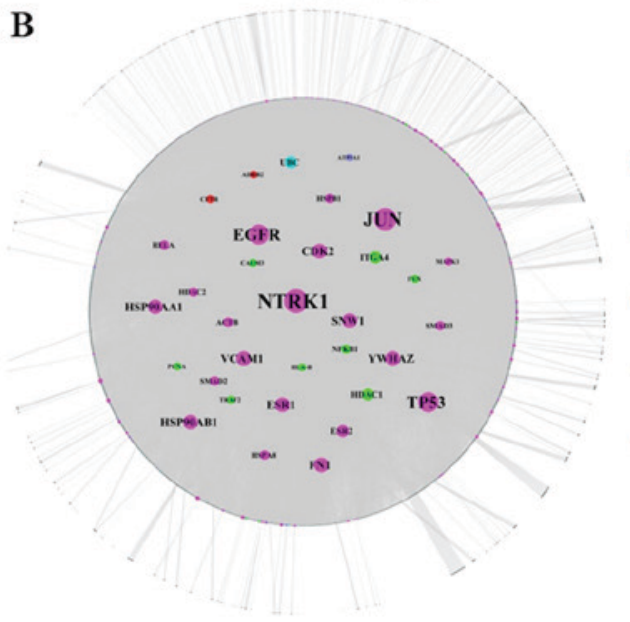

C
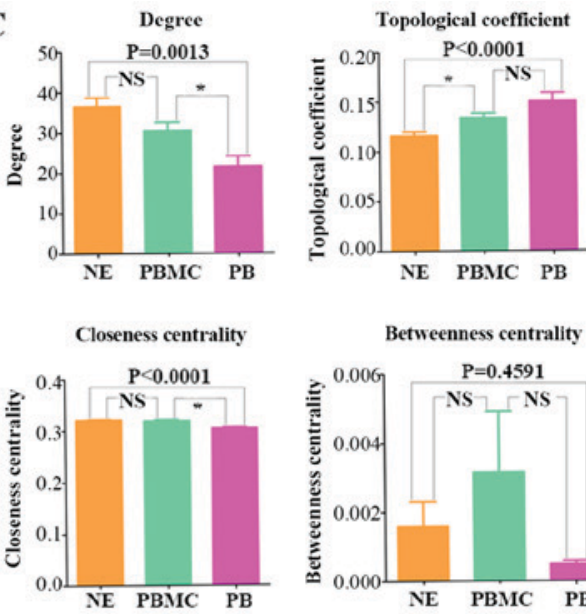

NE: nasal-epithelium

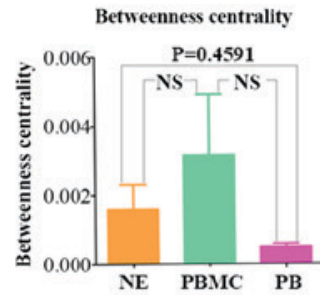

PB: peripheral blood

PBMC: peripheral blood mononuclear cells

D
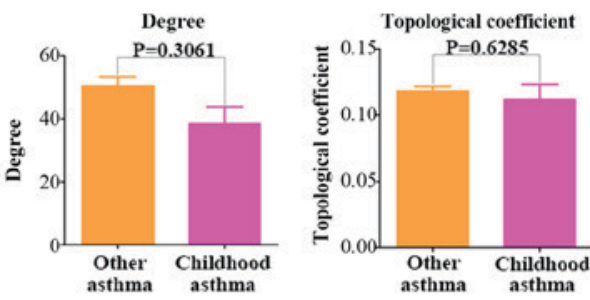

Childhood asthma non-DEG

Other protien
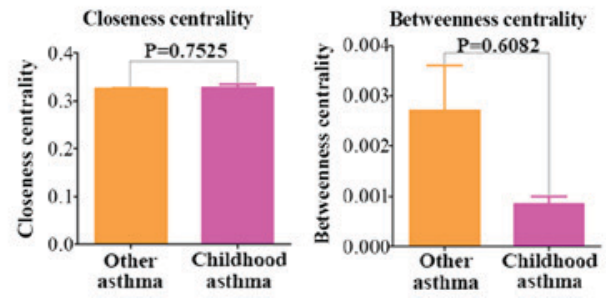

Figure 2. PPI network construction and analysis. (A) The PPI network of DEGs. (B) The PPI network of DEGs in the different tissues of asthmatic patients. (C) The topological property comparison of the DEGs between different tissues, including degree, topological coefficient, closeness centrality and betweenness centrality. (D) The topological property comparison between the DEGs of childhood asthmatic patients and non-childhood asthmatic patients. ${ }^{*} \mathrm{P}<0.05$, as indicated. PPI, protein-protein interaction; DEGs, differentially expressed genes; NS, not significant.

modules of black and green were significantly related to $\mathrm{NE}$ tissue; functional modules in the color of green and red were significantly associated with PBMC tissue.

The GO biological process (BP) enrichment analysis was performed to identify the function of DEGs associated with different tissue in asthmatic children (Fig. 4). The GO functional analysis results showed that the DEGs related to PB tissue were mainly enriched in the processes of acid secretion, sensory organ development and negative regulation of cell development, etc; the DEGs related to NE tissue were mainly involved in the processes of positive regulation of cell motility, and positive regulation of cellular component movement; the DEGs in PBMC tissue were mainly enriched in the processes of positive regulation of apoptotic process, and organ induction.

Construction of regulatory network. We constructed the regulatory network related to DEGs in different tissues (Fig. 5). As for the PBMC tissue, we took the interaction of DEGs in two databases to perform WGCNA analysis and the gene expression profile of GSE40732 dataset were used to analysis the adjust power.
The results showed that $F B X O 6$ was a hub gene in the regulatory network associated with $\mathrm{PB}$ tissue, which included 3,670 nodes and 5,330 relationship pairs (Fig. 5A). HDAC1 and cullin-7 (CUL7) were hub genes in the regulatory network of PBMC tissue, which included 6,803 nodes and 18,220 relationship pairs (Fig. 5B). Additionally, the regulatory network associated with NE tissue included 6,276 nodes and 18,209 relationship pairs, and amyloid $\beta$ precursor protein $(A P P)$ was a hub gene (Fig. 5C).

GO and KEGG pathway analyses of DEGs in regulatory network. The pathway analysis results showed that the DEGs in the PB tissue were mainly enriched in $M A P K$ signaling pathway, $P I 3 K$-Akt signaling pathway and neurotrophin signaling pathway, etc. (Fig. 6A). As for the DEGs in the PBMC tissue, the enriched pathways included neurotrophin signaling pathway, and insulin signaling pathway, etc. (Fig. 6B). The DEGs in the NE tissue were significantly enriched in ubiquitin mediated proteolysis, cell cycle and neurotrophin signaling pathway (Fig. 6C).

Moreover, the GO BP terms enriched by DEGs in PB tissue were mainly associated with cellular response to organic cyclic 

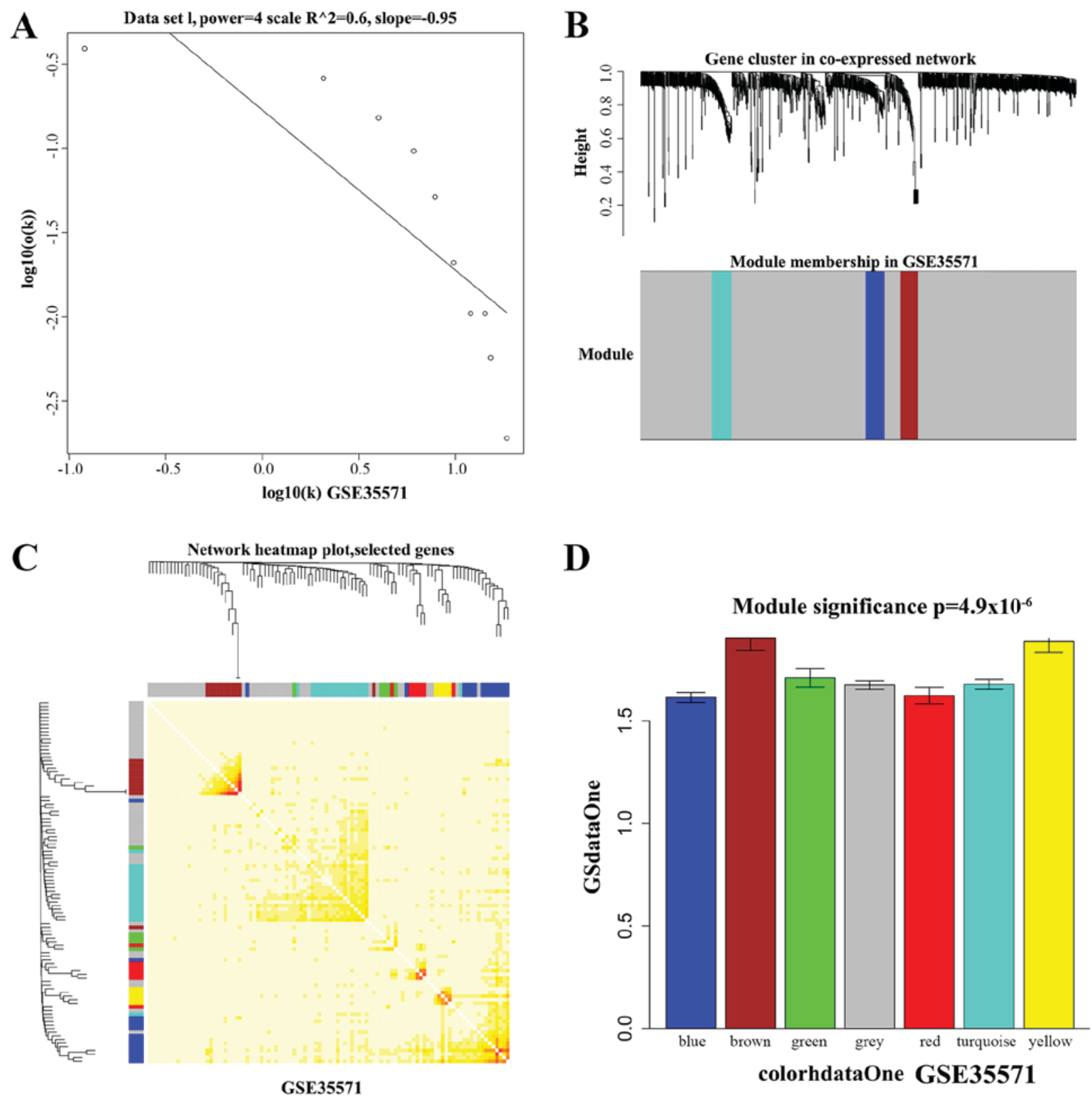

Figure 3. Gene co-expression modules analysis of GSE35571. (A) Soft threshold setting in the co-expression network. The x-axis represents gene degrees k, and the $y$-axis represents the ratio of genes $\mathrm{p}(\mathrm{k})$. (B) Hierarchical clustering heatmap of DEGs in co-expression network. (C) Hierarchical clustering heatmap showed the inner correlation between these function modules. (D) The GO enrichment analysis of DEGs in modules. The X-axis represents DEGs of modules and the $y$-axis represents the significance of GO analysis. DEGs, differentially expressed genes; GO, Gene Ontology.

compound (Fig. 7A). As for the DEGs in PBMC together with DEGs in NE tissue samples, the GO BP terms were related to cell cycle phase transition (Fig. 7B and C).

Specific DEGs in the regulatory network. The DEGs in the regulatory network were sequenced by $\mathrm{d} D R g$ values and top 20 DEGs were selected (Table II). The results showed that proliferating cell nuclear antigen (PCNA), integrin $\alpha-4$ (ITGA4), catenin $\alpha-1$ (CTNNA1), nuclear factor- $\kappa \mathrm{B} 1(N F-\kappa B 1)$ and mechanistic target of rapamycin $(M T O R)$ were specific DEGs that related to asthma. Besides, these DEGs were also identified in DisGetNET database associated with asthma. Our results indicated that $P C N A, I T G A 4, C T N N A 1, N F-\kappa B 1$ and MTOR might be the potential genes related to asthma in children.

\section{Discussion}

In the current study, we identified many DEGs from three types of tissue samples, including 1,662 DEGs from NE tissue samples, 572 DEGs from PB samples, and 146 DEGs from PBMC samples. In PPI network, FBXO6, HDACl and APP were hub genes and might play an important role in the process of asthma. In addition, PCNA, ITGA4, CTNNA1, NF- $\kappa B 1$ and $M T O R$ might be critical DEGs related to asthma in children.

FBXO6 encodes a member of the F-box protein family, which constitutes the subunit of ubiquitin protein ligase complex called SKP1-cullin-F-box (SCFS) (14). Overexpression of F-box protein FBXL19 can abrogate the inflammatory effects of $I L-33$ and lessen the severity of pulmonary inflammation in mouse models of pneumonia (15). However, the role 


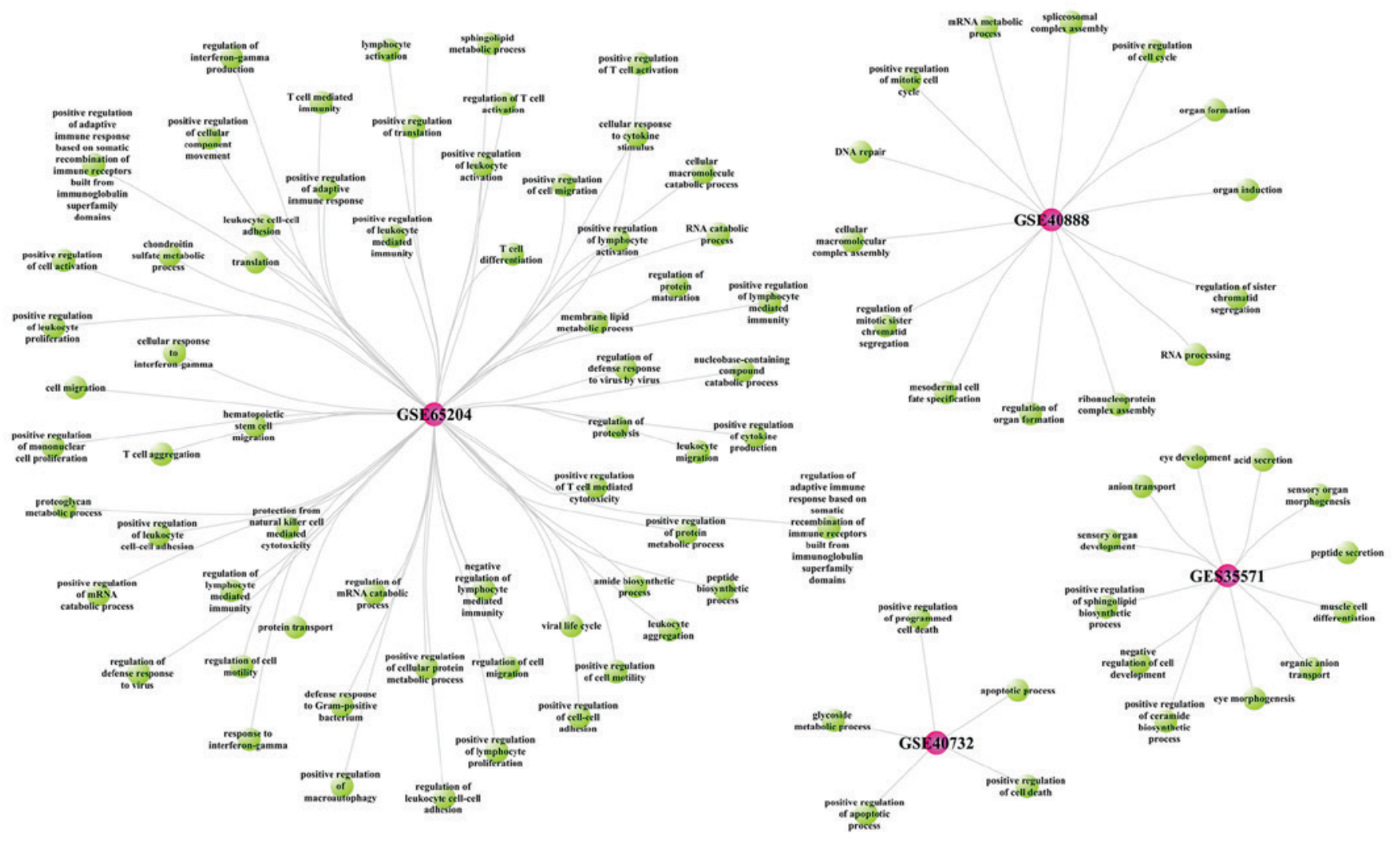

Figure 4. Gene Ontology Biological Process analysis of differentially expressed genes in different databases.

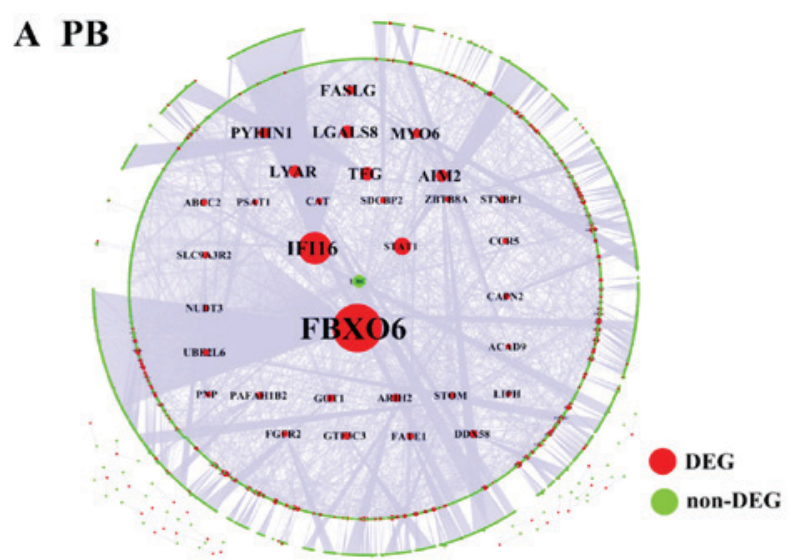

\section{B PBMC}

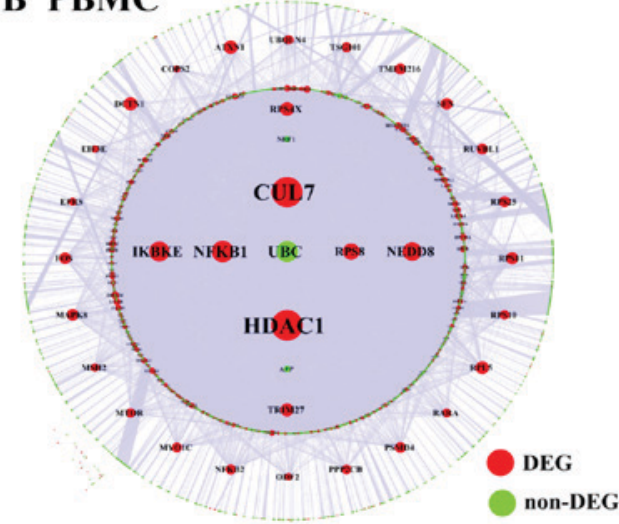

C NE

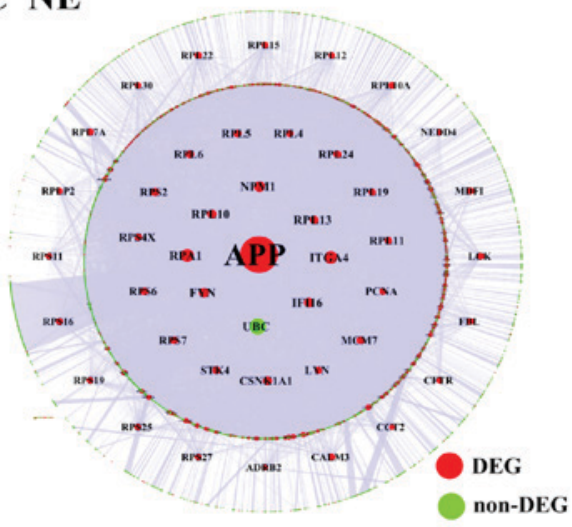

Figure 5. DEGs in the regulatory network associated with different types of tissues. (A) The DEGs in the regulatory network associated with PB. (B) The DEGs in the regulatory network associated with PBMC. (C) The DEGs in the regulatory network associated with NE tissue. DEGs, differentially expressed genes; $\mathrm{PB}$, peripheral blood; PBMC, peripheral blood mononuclear cells; NE, nasal-epithelium. 


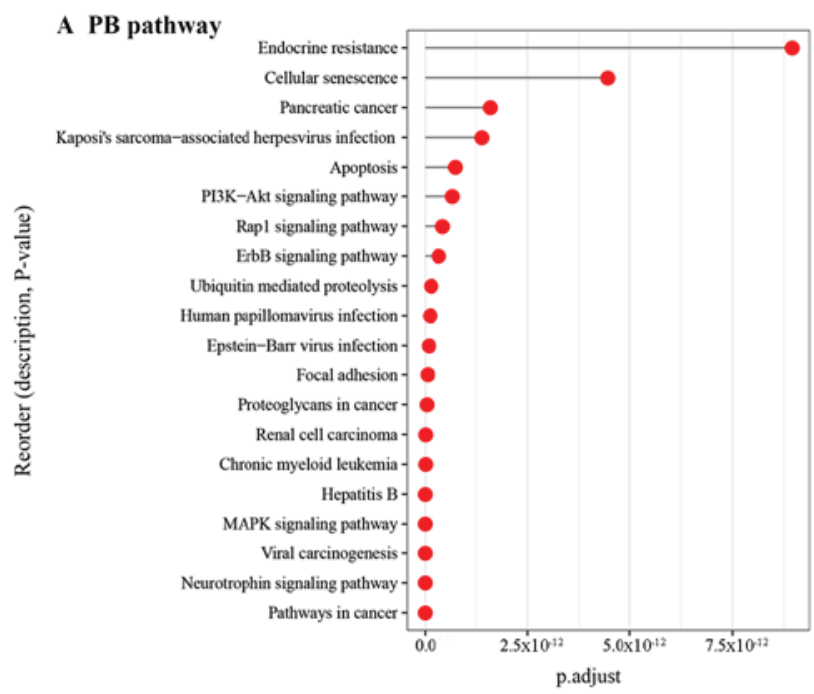

B PBMC pathway

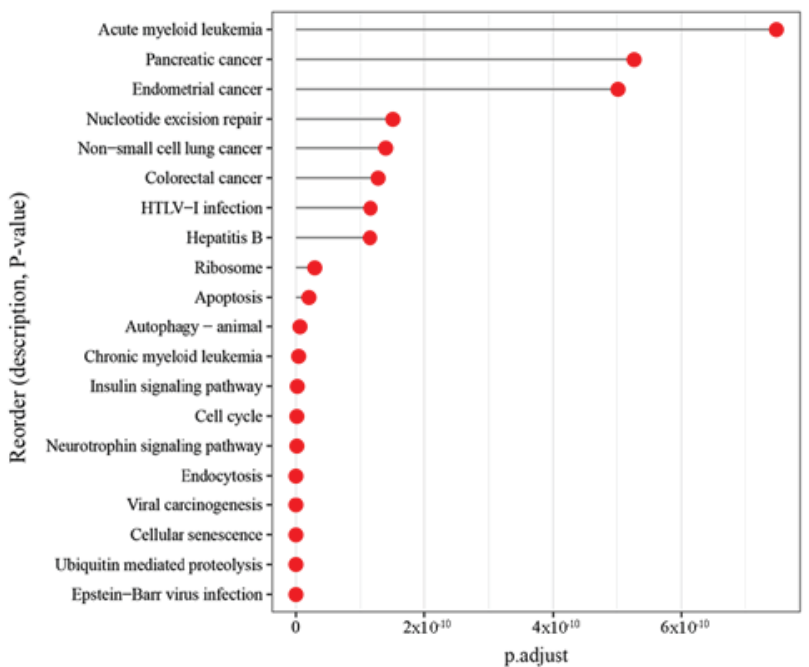

C NE pathway

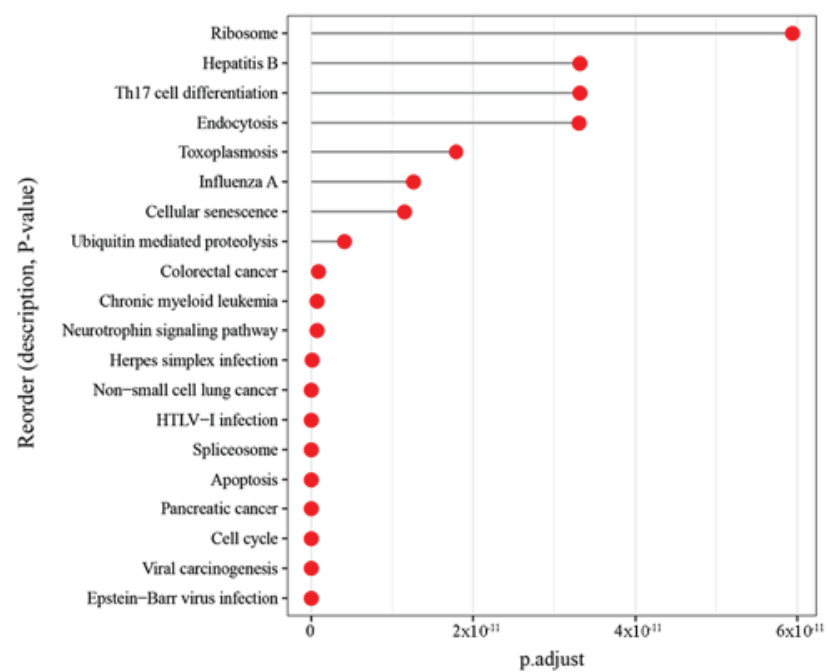

Figure 6. Kyoto Encyclopedia of Genes and Genomes pathway analysis for the DEGs in the regulatory network. (A) Pathway analysis for the DEGs in the network associated with PB. (B) Pathway analysis for the DEGs in the network associated with PBMC. (C) Pathway analysis for the DEGs in the network related to NE tissue. DEGs, differentially expressed genes; PB, peripheral blood; PBMC, peripheral blood mononuclear cells; NE, nasal-epithelium.

of FBXO6 in asthma has not been reported. In our study, we found that $F B X O 6$ were hub gene in the PPI network. Together with previous findings, we proposed that FBXO6 might relate to the inflammation in asthma.

HDAC1 is a member of HDAC family and highly expressed in inflammation-related diseases, such as arthritis (16). Deletion of HDACl increased allergic airway inflammation and promoted Th2 cytokine production in asthma mice, while asthmatic mice treated with herbal extract can resulted in significant anti-inflammatory and anti-allergic activity by increasing expression level of alveolar macrophages HDAC1 (17). Recent study showed that a polymorphism in the $H D A C l$ gene is associated with the response to corticosteroids in asthmatics (18). Our study revealed that $H D A C l$ was a hub gene in the PPI network and might play an important role in the process of asthma, which was consisting with previous studies. PCNA served as a factor to coordinate DNA replication and epigenetic inheritance, such as DNA methylation (19). A study showed that PCNA interacted with $H D A C l$ in human cells in vitro can co-localize in the cell nucleus, and finally led to integration of DNA replication (20). DNA methylation changes in PB were associated with childhood allergic asthma (9). These finding indicated that PCNA interacted with $H D A C 1$ might involve in DNA methylation of asthma in children. In addition, a previous study showed that PCNA expression was associated with the epithelium thickness in corticosteroid-dependent asthma (21). The cell proliferation related molecule $P C N A$ and cell activation related molecule $N F-\kappa B$ were both highly expressed in corticosteroid-dependent asthmatic subjects (21), revealing the potential role in the treatment and disease epithelium repair.

$I T G A 4$ is also named as $C D 49 d$. A study reported that up-regulation of lysophosphatidic acid receptor 1 and down-regulation of ITGA4 can increase the number of monocytes in the PB and finally impact on immune function (22). However, the relationships between ITGA4 and asthma disease were unclear. The PPI network analysis in our study revealed that ITGA4 might be a major factor related to asthma in children. CTNNA1 is also known as $\alpha$ E-catenin, and it plays a major role in epithelial tissue, both at adherent junctions and 

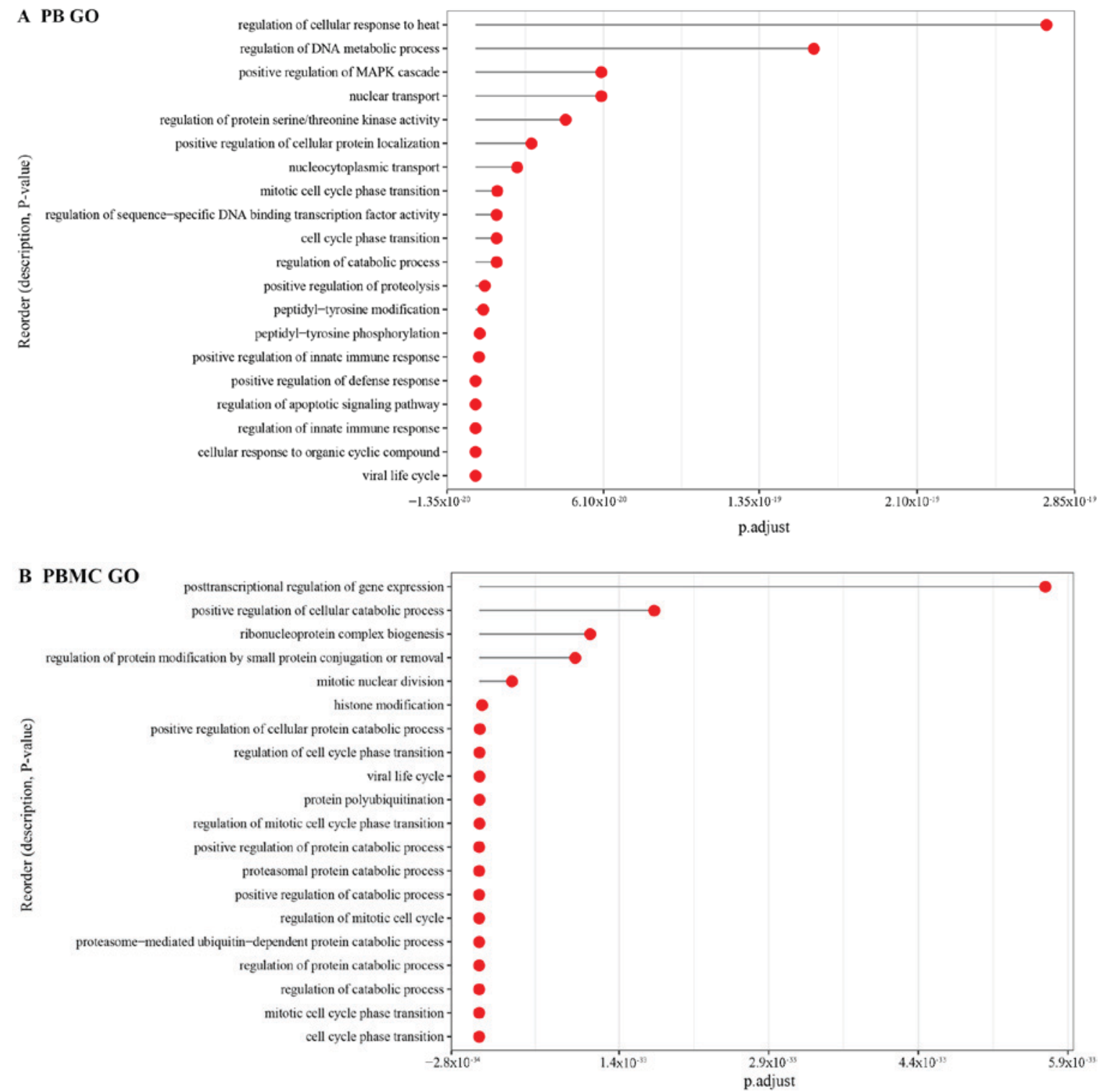

\section{NE GO}

RNA splicing, via transesterification reactions with bulged adenosine as nucleophileproteasome-mediated ubiquitin-dependent protein catabolic processregulation of protein catabolic process RNA splicing viral transcription mRNA processing -

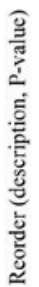

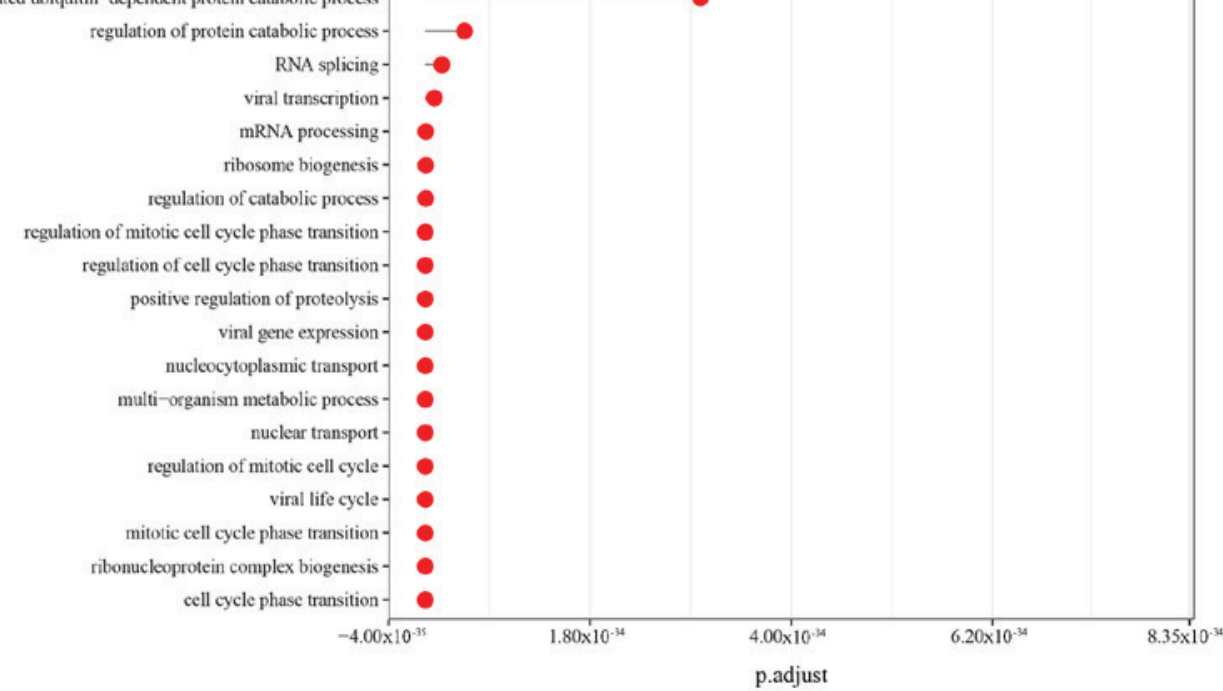

Figure 7. GO BP analysis for the DEGs in the regulatory network. (A) GO BP analysis for the DEGs in the regulatory network associated with PB. (B) GO $\mathrm{BP}$ analysis for the DEGs in the regulatory network associated with PBMC. (C) GO BP analysis for the DEGs in the regulatory network associated with NE tissue. GO, Gene Ontology; BP, biological process; DEGs, differentially expressed genes; PB, peripheral blood; PBMC, peripheral blood mononuclear cells; NE, nasal-epithelium. 
Table II. Top 20 nodes in the regulatory network associated with the DEGs of asthmatic children.

\begin{tabular}{|c|c|c|c|c|c|c|c|c|c|}
\hline \multirow[b]{2}{*}{ No. } & \multicolumn{3}{|c|}{ NE Samples } & \multicolumn{3}{|c|}{ PB Samples } & \multicolumn{3}{|c|}{ PBMC samples } \\
\hline & DEG & $\mathrm{dDRg}$ & Label & DEG & $\mathrm{dDRg}$ & Label & DEG & $\mathrm{dDRg}$ & Label \\
\hline 1 & APP & 202.5712 & No & FBXO6 & 42.82477 & No & $\mathrm{HDAC}^{\mathrm{a}}{ }^{\mathrm{a}}$ & 106.7973 & Other asthma \\
\hline 2 & SETDB1 & 78.09696 & No & ARIH2 & 36.51952 & No & RPS8 & 61.2846 & No \\
\hline 3 & RPA1 & 68.47313 & No & IFI16 & 33.23794 & No & RPS11 & 58.52655 & No \\
\hline 4 & STK4 & 66.04559 & No & PAFAH1B2 & 30.54515 & No & RPS4X & 39.92426 & No \\
\hline 5 & NEDD4 & 61.91617 & No & TFG & 29.83253 & No & TRIM27 & 39.09394 & No \\
\hline 6 & CSNK1A1 & 57.83066 & No & FGFR2 & 26.67677 & No & RPL5 & 35.9798 & No \\
\hline 7 & EDC4 & 53.42212 & No & LGALS8 & 26.21989 & No & CUL7 & 29.2522 & No \\
\hline 8 & CTDP1 & 49.7963 & No & NEK6 & 24.95025 & No & NEDD8 & 28.72204 & No \\
\hline 9 & RPS2 & 43.28688 & No & LYAR & 22.42635 & No & RPS10 & 27.68076 & No \\
\hline 10 & CALM3 & 41.95928 & No & SPINT2 & 19.52418 & No & RPS25 & 27.62278 & No \\
\hline 11 & RPS7 & 40.06917 & No & GOT1 & 17.31375 & No & TMEM216 & 27.26486 & No \\
\hline 12 & IFI16 & 39.24005 & No & MYO6 & 17.17874 & No & $\mathrm{NFKB}^{\mathrm{a}}{ }^{\mathrm{a}}$ & 25.80718 & Other asthma \\
\hline 13 & MID2 & 35.10338 & No & PELO & 16.25726 & No & RPL35A & 25.62394 & No \\
\hline 14 & $\mathrm{PCNA}^{\mathrm{a}}$ & 32.54975 & Other asthma & PTK6 & 15.76188 & No & PSMD4 & 25.04536 & No \\
\hline 15 & RPL10 & 30.89499 & No & CTNNA1 $^{\mathrm{a}}$ & 15.75888 & Other asthma & EIF3E & 19.85108 & No \\
\hline 16 & RPS16 & 29.85522 & No & NTRK2 & 14.60812 & No & MTOR $^{a}$ & 19.81246 & Other asthma \\
\hline 17 & ITGA4 $^{\mathrm{a}}$ & 29.2464 & Other asthma & LIPH & 12.77759 & No & DDX24 & 19.53866 & No \\
\hline 18 & MCM7 & 28.55749 & No & CA10 & 12.35675 & No & UBQLN4 & 19.34685 & No \\
\hline 19 & SPTAN1 & 28.18446 & No & NUDT3 & 11.95574 & No & ATXN1 & 18.88822 & No \\
\hline 20 & LZTS2 & 28.04549 & No & SLC9A3R2 & 11.93033 & No & IKBKE & 17.33138 & No \\
\hline
\end{tabular}

The dDRg values of DEGs in the regulatory network are presented. aDEGs associated with asthma. DEGs, differentially expressed genes; PB, peripheral blood; dDRg, weighted control values; PBMC, peripheral blood mononuclear cells; NE, nasal-epithelium.

in signaling pathways (23). Epithelial damage from airway inflammation during asthma may result in immune response to self-antigens, including $\alpha E$-catenin and epidermal group factor receptor $(E G F R)$; and finally contributes to the pathogenesis of asthma (24). Moreover, a replication study in a Caucasian worker population revealed that $\alpha$-catenin gene variants were also associated with diisocyanate asthma (25). These results suggested that CTNNA1 might be critical gene that regulated the progressive of asthma in children.

MTOR is a serine/threonine kinase that is evolutionary conserved and can regulate lymphocyte cellular immunity by activating cytokine secreted from inflammatory cells (26). $M T O R C 2$ regulated the differentiation of naive $\mathrm{CD} 4+\mathrm{T}$ cells into Th9 cells, and mTORC2 deficiency in T cells could result in less severe inflammation in the murine allergic airway inflammation model (27). Zhang et al (28) revealed that increased serum $m$ TOR pathway activation can lead to elevated levels of Th17 cells and IL-4, following decreased Treg cells and IFN- $\gamma$. Our study demonstrated a clear relationship between MTOR in the PBMC of childhood patients with allergic asthma. These findings strongly suggested a necessary for $m T O R$ pathway activation in asthma process. In addition, $N F-\kappa B l$ is reported as a transcription factor that is activated by multiple intra-cellular and extra-cellular stimuli such as cytokines, oxidant-free radicals, and bacterial or viral products (29). Activated $N F-\kappa B$ can stimulate the expression of genes involved in many biological or pathological processes, including acute lung injury/acute respiratory distress syndrome and asthma. Recent study showed that the over-expression of $P I 3 K$ and $N F-\kappa B$ in childhood asthma were negatively correlated with pulmonary functions, which indicated that $P I 3 \mathrm{~K}$ and $N F-\kappa B$ might involve in the development of bronchial asthma in children (30). Inhibition of the $N F-\kappa B$ signaling pathway can improve airway inflammation in an ovalbumin-induced rat model (31). In our study, we also found that the $N F-\kappa B l$ and $m T O R$ were hub genes related to asthma in children. Considering the previous studies, we suggested that $N F-\kappa B l$ interacted with $m T O R$ might play an important role in the progression of asthma in children, such as inflammation in asthma. Additionally, a study of asthmatic mouse model indicated that mTOR is activated during asthma onset and inhibited during asthma remission, and blocking the mTOR pathway in asthmatic mice restores the Th17/Treg and Th1/Th2 cytokines balances (28). These findings strongly documented a critical role of mTOR pathway activation in asthma onset, revealing potential targets for asthma treatments.

Using the network analysis, we also identified $A P P$ hitherto not associated with asthma as important hub gene in regulatory network associated with NE tissue. APP gene encoding the amyloid beta precursor protein is known as a major player in Alzheimer's disease (AD) that have immune and inflammatory components (32). Recent studies also suggested a link between $A P P$ and asthma genes $(32,33)$. It is revealed that $A P P$ 
was potentially associated with airway hyperresponsiveness through the interaction with a disintegrin and metalloproteinase (ADAM33) (33). ADAM33 is an asthma susceptibility gene with catalytic properties, functioned as a negative regulator of $A P P$ (34). In our module, $A P P$ was found potentially interacted with other asthma genes, it is speculated that these possible connections can provide new insights for exploring the functional role and relationships of these genes in asthma, as well as in AD.

In conclusion, our findings suggest that genes are differentially expressed in the three type tissue samples of asthmatic children, including NE, PB and PBMC samples. Among these DEGs, FBXO6, HDAC1 and APP interact with PCNA, ITGA4, $C T N N A 1, N F-\kappa B 1$ and $m T O R$ might be critical DEGs related to asthma in children.

\section{Acknowledgements}

Not applicable.

\section{Funding}

The present study was supported by The Science and Technology Development Fund of Fengxian District, Shanghai (grant no. 20151236).

\section{Availability of data and materials}

All data generated or analyzed during this study are included in this published article.

\section{Authors' contributions}

GW and CW conceived and designed the present study. $\mathrm{CW}, \mathrm{HL}$ and LC conducted the data analysis. CW and GW prepared the manuscript. All of the authors reviewed the manuscript.

\section{Ethics approval and consent to participate}

Not applicable.

\section{Consent for publication}

Not applicable.

\section{Competing interests}

The authors declare that they have no competing interests.

\section{References}

1. U.S. Department of Health and Human Services, Centers for Disease Control and Prevention and National Center for Health Statistics: Tables of Summary Health Statistics: National Health Interview Survey. Table C-1b, pp3-4, 2016.

2. James CV and Rosenbaum S: Paying for quality care: Implications for racial and ethnic health disparities in pediatric asthma. Pediatrics 123 (Suppl 3): S205-S210, 2009.

3. Lin HW and Lin SC: Environmental factors association between asthma and acute bronchiolitis in young children-a perspective cohort study. Eur J Pediatr 171: 1645-1650, 2012.
4. Martinez FD: Genes, environments, development and asthma: A reappraisal. Eur Respir J 29: 179-184, 2007.

5. Goetghebuer T, Isles K, Moore C, Thomson A, Kwiatkowski D and Hull J: Genetic predisposition to wheeze following respiratory syncytial virus bronchiolitis. Clin Exp Allergy 34: 801-803, 2004.

6. Finkelstein Y, Bournissen FG, Hutson JR and Shannon M: Polymorphism of the ADRB2 gene and response to inhaled beta-agonists in children with asthma: A meta-analysis. J Asthma 46: 900-905, 2009.

7. Wills-Karp M: Immunologic basis of antigen-induced airway hyperresponsiveness. Annu Rev Immunol 17: 255-281, 1999.

8. Yang IV, Pedersen BS, Liu AH, O'Connor GT, Pillai D, Kattan M, Misiak RT, Gruchalla R, Szefler SJ, Khurana Hershey GK, et al: The nasal methylome and childhood atopic asthma. J Allergy Clin Immunol 139: 1478-1488, 2017.

9. Yang IV, Pedersen BS, Liu A, O'Connor GT, Teach SJ, Kattan M, Misiak RT, Gruchalla R, Steinbach SF, Szefler SJ, et al: DNA methylation and childhood asthma in the inner city. J Allergy Clin Immunol 136: 69-80, 2015.

10. Raedler D, Ballenberger N, Klucker E, Böck A, Otto R, Prazeres da Costa O, Holst O, Illig T, Buch T, von Mutius E and Schaub B: Identification of novel immune phenotypes for allergic and nonallergic childhood asthma. J Allergy Clin Immunol 135: 81-91, 2015

11. Williams-DeVane CR, Reif DM, Hubal EC, Bushel PR, Hudgens EE, Gallagher JE and Edwards SW: Decision tree-based method for integrating gene expression, demographic, and clinical data to determine disease endotypes. BMC Syst Biol 7: 119, 2013.

12. Ritchie ME, Phipson B, Wu D, Hu Y, Law CW, Shi W and Smyth GK: Limma powers differential expression analyses for RNA-sequencing and microarray studies. Nucleic Acids Res 43: e47, 2015 .

13. Langfelder $\mathrm{P}$ and Horvath S: WGCNA: An R package for weighted correlation network analysis. BMC Bioinformatics 9: 559, 2008.

14. Chen X, Duan LH, Luo PC, Hu G, Yu X, Liu J, Lu H and Liu B: FBXO6-mediated ubiquitination and degradation of Ero1L inhibits endoplasmic reticulum stress-induced apoptosis. Cell Physiol Biochem 39: 2501-2508, 2016.

15. Zhao J, Wei J, Mialki RK, Mallampalli DF, Chen BB, Coon T, Zou C, Mallampalli RK and Zhao Y: F-box protein FBXL19-mediated ubiquitination and degradation of the receptor for IL-33 limits pulmonary inflammation. Nat Immunol 13: 651-658, 2012.

16. Cantley MD, Fairlie DP, Bartold PM, Marino V, Gupta PK and Haynes DR: Inhibiting histone deacetylase 1 suppresses both inflammation and bone loss in arthritis. Rheumatology (Oxford) 54: 1713-1723, 2015.

17. Grausenburger R, Bilic I, Boucheron N, Zupkovitz G, El-Housseiny L, Tschismarov R, Zhang Y, Rembold M, Gaisberger M, Hartl A, et al: Conditional deletion of histone deacetylase 1 in T cells leads to enhanced airway inflammation and increased Th2 cytokine production. J Immunol 185: 3489-3497, 2010.

18. Kim MH, Kim SH, Kim YK, Hong SJ, Min KU, Cho SH and Park HW: A polymorphism in the histone deacetylase 1 gene is associated with the response to corticosteroids in asthmatics. Korean J Intern Med 28: 708-714, 2013.

19. Chuang LS, Ian HI, Koh TW, Ng HH, Xu G and Li BF: Human DNA-(cytosine-5) methyltransferase-PCNA complex as a target for p21WAF1. Science 277: 1996-2000, 1997.

20. Milutinovic S, Zhuang Q and Szyf M: Proliferating cell nuclear antigen associates with histone deacetylase activity, integrating DNA replication and chromatin modification. J Biol Chem 277: 20974-20978, 2002.

21. Vignola AM, Chiappara G, Siena L, Bruno A, Gagliardo R, Merendino AM, Polla BS, Arrigo AP, Bonsignore G, Bousquet J and Chanez P: Proliferation and activation of bronchial epithelial cells in corticosteroid-dependent asthma. J Allergy Clin Immunol 108: 738-746, 2001.

22. Maugeri N, Powell JE, t Hoen PA, de Geus EJ, Willemsen G, Kattenberg M, Henders AK, Wallace L, Penninx B, Hottenga JJ, et al: LPAR1 and ITGA4 regulate peripheral blood monocyte counts. Hum Mutat 32: 873-876, 2011.

23. Herrenknecht K, Ozawa M, Eckerskorn C, Lottspeich F, Lenter M and Kemler R: The uvomorulin-anchorage protein alpha catenin is a vinculin homologue. Proc Natl Acad Sci USA 88: 9156-9160, 1991. 
24. Liu M,Subramanian V,Christie C, Castro M and Mohanakumar T: Immune responses to self-antigens in asthma patients: Clinical and immunopathological implications. Hum Immunol 73: 511-516, 2012.

25. Bernstein DI, Kashon M, Lummus ZL, Johnson VJ, Fluharty K, Gautrin D, Malo JL, Cartier A, Boulet LP, Sastre J, et al: CTNNA3 ( $\alpha$-catenin) gene variants are associated with diisocyanate asthma: A replication study in a Caucasian worker population. Toxicol Sci 131: 242-246, 2013.

26. Wullschleger $S$, Loewith $R$ and Hall MN: TOR signaling in growth and metabolism. Cell 124: 471-484, 2006.

27. Chen H, Zhang L, Wang P, Su H, Wang W, Chu Z, Zhang L, Zhang $\mathrm{X}$ and Zhao Y: mTORC2 controls Th9 polarization and allergic airway inflammation. Allergy 72: 1510-1520, 2017.

28. Zhang Y, Jing Y, Qiao J, Luan B, Wang X, Wang L and Song Z: Activation of the mTOR signaling pathway is required for asthma onset. Sci Rep 7: 4532, 2017.

29. Meyer R, Hatada EN, Hohmann HP, Haiker M, Bartsch C, Röthlisberger U, Lahm HW, Schlaeger EJ, van Loon AP and Scheidereit C: Cloning of the DNA-binding subunit of human nuclear factor kappa B: The level of its mRNA is strongly regulated by phorbol ester or tumor necrosis factor alpha. Proc Natl Acad Sci USA 88: 966-970, 1991.
30. Shi HL, Liu JB and Lu AP: Expression profiles of PI3K, NF- $\kappa$ B, and STAT1 in peripheral blood mononuclear cells in children with bronchial asthma. Zhongguo Dang Dai Er Ke Za Zhi 18: 614-617, 2016 (In Chinese).

31. Li Z, Zheng J, Zhang N and Li C: Berberine improves airway inflammation and inhibits NF- $\kappa \mathrm{B}$ signaling pathway in an ovalbumin-induced rat model of asthma. J Asthma 53: 999-1005, 2016.

32. Liu Y, Brossard M, Sarnowski C, Vaysse A, Moffatt M, Margaritte-Jeannin P, Llinares-López F, Dizier MH, Lathrop M, Cookson W, et al: Network-assisted analysis of GWAS data identifies a functionally-relevant gene module for childhood-onset asthma. Sci Rep 7: 938, 2017.

33. Vishweswaraiah S, Veerappa AM, Mahesh PA, Jayaraju BS, Krishnarao CS and Ramachandra NB: Molecular interaction network and pathway studies of ADAM33 potentially relevant to asthma. Ann Allergy Asthma Immunol 113: 418-424.e1, 2014.

34. Zou J, Zhu F, Liu J, Wang W, Zhang R, Garlisi CG, Liu YH, Wang S, Shah H, Wan Y and Umland SP: Catalytic activity of human ADAM33. J Biol Chem 279: 9818-9830, 2004.

This work is licensed under a Creative Commons Attribution-NonCommercial-NoDerivatives 4.0 International (CC BY-NC-ND 4.0) License. 